\title{
Obstrucción intestinal por cápsula endoscópica en un paciente con enfermedad de Crohn
}

\section{Bowel obstruction due to video capsule endoscopy in a patient with Crohn's disease}

\author{
Guillermo R. Guevara-Morales ${ }^{1 *}$ y Juan C. Castellanos-Juárez ${ }^{2}$ \\ ${ }^{1}$ Servicio de Cirugía General; ${ }^{2}$ Servicio de Coloproctología. Hospital Regional de Veracruz, Instituto de Seguridad y Servicios Sociales de los
} Trabajadores del Estado (ISSSTE), Veracruz, Veracruz, México

\section{Resumen}

Antecedentes: La retención de la cápsula endoscópica (CE) es una complicación grave y provoca de forma excepcional obstrucción intestinal aguda en su presentación. Caso clínico: Paciente masculino de 64 años, tratado previamente por gastroenterología por enfermedad de Crohn. Muestra buena respuesta inicial al tratamiento farmacológico por seis semanas, pero después refiere dolor y síntomas no explicados por la colonoscopia. Se realizó CE, con aparición a las 48 horas de cuadro de obstrucción intestinal, signos de respuesta inflamatoria sistémica e irritación peritoneal. Se practicó laparotomía exploradora más resección intestinal con anastomosis laterolateral mecánica y el informe de histopatología confirmó obstrucción de la luz intestinal por CE impactada en la zona de estenosis del íleon terminal. En el postoperatorio inmediato la evolución es buena. A las ocho semanas del procedimiento acude por fístula enterocutánea que tuvo buena respuesta al tratamiento conservador. Conclusión: La tasa de retención de CE en la enfermedad de Crohn es de 5 a 6\%; antes de programarla se recomienda realizar estudios de imagen para evaluar la permeabilidad de la luz intestinal, si bien los estudios negativos no excluyen la presencia de estenosis. En los casos en que esté indicada la resección intestinal, se recomienda que ésta sea amplia con anastomosis laterolateral mecánica para reducir el riesgo de recurrencia de la enfermedad.

PALABRAS CLAVE: Obstrucción intestinal. Cápsula endoscópica. Enfermedad de Crohn.

\begin{abstract}
Background: The retention of the endoscopic capsule $(E C)$ is a serious complication and exceptionally, acute intestinal obstruction is conditioned. Clinical case: 64 years-old man, previously treated by gastroenterology for Crohn's disease. With good initial response to pharmacological treatment for 6 weeks, subsequently with the onset of pain and symptoms not explained by colonoscopy. EC was performed, presenting at 48 hours bowel obstruction symptoms with data of systemic inflammatory response and peritoneal irritation. Exploratory laparotomy with bowel resection and side-to-side stapled anastomosis was performed, histopathology report confirmed obstruction of the bowel lumen due to impacted EC in ileal stenosis area. In the immediate postoperative period with good evolution. At 8 weeks of the procedure, he went for an enterocutaneous fistula, which had a good response to conservative treatment. Conclusion: The rate of retention of CE in Crohn's disease is 5-6\%, prior to its use, it is recommended to perform imaging studies to evaluate the bowel permeability, however negative studies do not exclude the presence of stenosis. In cases where intestinal resection is indicated, it is recommended to be wide with side-to-side stapled anastomosis to reduce the risk of recurrence of the disease.
\end{abstract}

KEY WORDS: Bowel obstruction. Capsule endoscopy. Crohn's disease.

\author{
Correspondencia: \\ *Guillermo R. Guevara-Morales \\ Avda. Díaz Mirón, esquina Sayula, s/n \\ Fracc. Moderno \\ C.P. 91918, Veracruz, Ver., México \\ E-mail: grobertoguevara@gmail.com
}

Fecha de recepción: 07-09-2018

Fecha de aceptación: 20-02-2019

DOI: 10.24875/CIRU.19000710
Cir Cir. 2019;87(S1):48-52

Contents available at PubMed www.cirugiaycirujanos.com 


\section{Introducción}

La cápsula endoscópica (CE) es una de las herramientas con mayor utilidad para la evaluación de afecciones del intestino delgado. La retención de la CE es la complicación más temida de este método diagnóstico. Existen diversas causas de retención de la CE, como enteropatía por AINE, tumores de intestino delgado, enteritis por radiación, estenosis de anastomosis previas y enfermedad de Crohn.

La retención de la CE puede presentarse sin compromiso del tránsito intestinal o con obstrucción intestinal, la cual se presenta de forma excepcional de manera aguda. Se calcula que ocurre en 1 a $2 \%$ de los casos en los que se utiliza este método diagnóstico, si bien se ha notificado que en trastornos con riesgo de estenosis puede ser hasta de 13\%1,2.

En los pacientes con diagnóstico de enfermedad de Crohn, el uso de la cápsula endoscópica se justifica por la presencia de síntomas que no pueden explicar la ileocolonoscopia o los estudios de imagen, por la sospecha de recurrencia después de una operación con colonoscopia negativa, u otros factores ${ }^{3}$ (Tabla 1).

Se calcula que la tasa de retención de la $\mathrm{CE}$ en pacientes con enfermedad de Crohn es de 5 a $6 \%$, aunque algunos estudios sugieren que la tasa podría ser más alta. Se presenta un caso clínico de obstrucción intestinal aguda por cápsula endoscópica en un paciente con enfermedad de Crohn.

\section{Caso clínico}

Paciente masculino de 64 años de edad fumador crónico con antecedentes de osteoartritis y uveítis en tratamiento y colecistectomía cinco años antes de su ingreso. Se lo refiere de modo inicial al Servicio de gastroenterología por cuadro de dolor abdominal, náusea, vómito, distensión abdominal, diarrea crónica intermitente tratada con loperamida y hemorragia de tubo digestivo bajo (tipo hematoquecia) de tres años de evolución.

Se realizaron colonoscopia y toma de biopsias, con informe de úlceras y disminución del calibre de la luz a nivel de colon ascendente y úlceras con retracción de tejido en el plano de la válvula ileocecal. Las biopsias con informe histopatológico confirmaron enfermedad de Crohn.

Se inició tratamiento con budesonida (9 mg/día) y se logró la remisión de los síntomas en seis semanas. Sin embargo, con posterioridad se presentaron inicio de dolor abdominal difuso y persistencia de éste de
Tabla 1. Recomendaciones del uso de la cápsula endoscópica en la enfermedad de Crohn*

\begin{tabular}{ll}
\hline Condición clínica & Grado de Recomendación \\
\hline 1. Pacientes que presentan & Recomendación fuerte \\
características clínicas & \\
consistentes con enfermedad & \\
de Crohn e ileocolonoscopia y & \\
estudios de imagen negativos &
\end{tabular}

2. Pacientes con enfermedad de Recomendación fuerte Crohn y características clínicas no explicadas en los hallazgos de la ileocolonoscopia o estudios de imagen

3. Pacientes con enfermedad de Crohn cuando se intenta evaluar la cicatrización de la mucosa del intestino delgado (que no está al alcance de la ileocolonoscopia)*

\section{Pacientes con sospecha de recurrencia de la enfermedad de Crohn en intestino delgado después de alguna colectomía, no diagnosticada en la ileocolonoscopia o estudios de imagen}

5. Pacientes con dolor abdominal crónico o diarrea como únicos síntomas y sin evidencia de biomarcadores relacionados con enfermedad de Crohn. Sugerencia en contra del uso de la cápsula endoscópica para el diagnóstico de la enfermedad de Crohn.

*Resumen de las recomendaciones del Consenso y guía de práctica clínica del uso de la cápsula endoscópica de la American Gastroenterological Association (AGA) del $2017^{3}$.

forma intermitente (8/10 en la escala analógica del dolor), sin alteraciones del tránsito intestinal. Se indicó cápsula endoscópica para valorar el resto del tracto digestivo por parte del Servicio de gastroenterología. A las 24 horas tras la introducción de la CE, el paciente experimentó dolor abdominal y distensión abdominal, sin datos de respuesta inflamatoria sistémica en ese momento valorado por el Servicio de cirugía general, que indicó tratamiento conservador. Durante su evolución persistió con dolor abdominal y su exacerbación; en la valoración por el Servicio de cirugía de colon y recto, efectuada a las 48 horas luego de la ingestión de la CE, el paciente se encontró clínicamente taquicárdico con abdomen no peristáltico, distendido, timpánico y signos de irritación peritoneal. Los estudios de laboratorio mostraron evidencia de leucocitosis $\left(14.5 \times 10^{3}\right)$ con desviación a la izquierda; las radiografías de abdomen proporcionaron imágenes indicativas de dilatación de asas de 


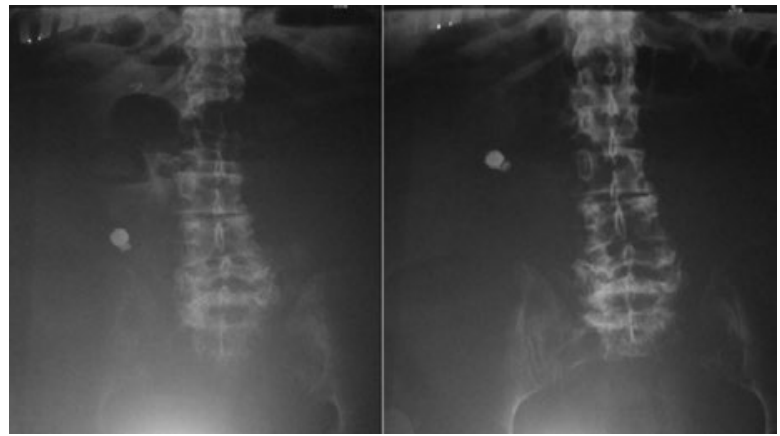

Figura 1. Radiografías simples de abdomen en bipedestación y decúbito que muestran dilatación de las asas de intestino delgado, niveles hidroaéreos, así como imagen radiopaca que corresponde a la cápsula endoscópica.

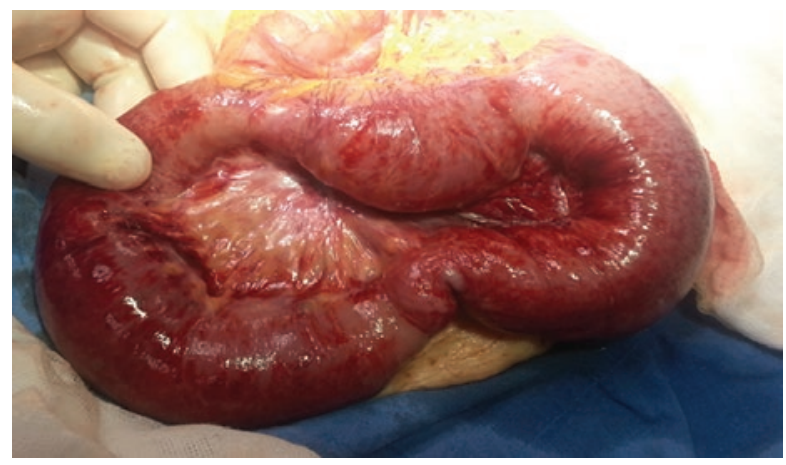

Figura 2. Íleon con segmentos dilatados, serosa con eritema, cambios en el patrón vascular y zonas con disminución del diámetro intestinal.

intestino delgado, niveles hidroaéreos y retención de $\mathrm{CE}$, razón por la cual se propuso laparotomía exploradora (Fig. 1).

Se realizó laparotomía exploradora y se identificó distensión generalizada de asas de intestino delgado en íleon con zonas de diminución del diámetro intestinal y datos macroscópicos indicativos de inflamación aguda y crónica (Fig. 2). En el íleon, a $110 \mathrm{~cm}$ de la válvula ileocecal, se reconoció un área de estenosis concéntrica y se palpó la CE impactada (Fig. 3). Se efectuó resección de $60 \mathrm{~cm}$ del segmento afectado y luego se creó anastomosis intestinal laterolateral con engrapadora mecánica tipo GIA de $75 \mathrm{~mm}$. Se constataron la permeabilidad y la ausencia de fuga de la anastomosis. El estudio histopatológico informó lo siguiente: pieza quirúrgica de íleon con disminución de la luz secundaria a proceso fibroestenótico, con obstrucción de la luz por cápsula endoscópica impactada en la zona de estenosis de unos $6 \mathrm{~cm}$ y signos de enfermedad de Crohn activa. El informe de la cápsula endoscópica reveló úlceras y divertículos en yeyuno y datos de retención con falla de paso al colon (Fig. 4).

Durante el postoperatorio (PO) inmediato, el paciente tenía adecuada evolución; se descartó fuga de la

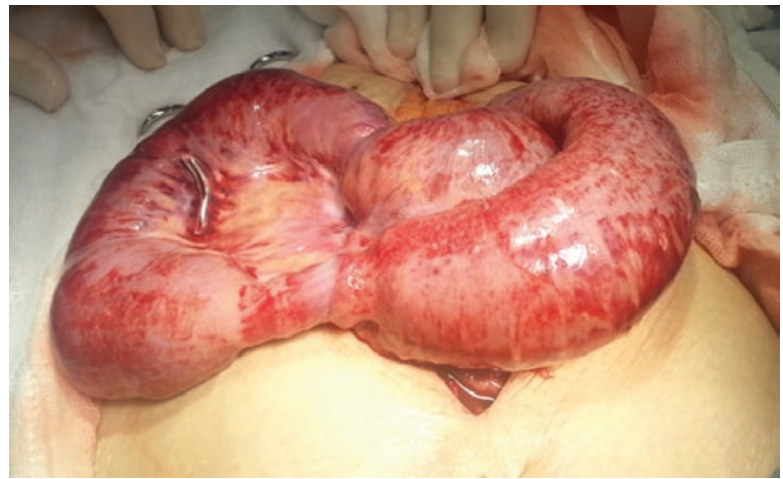

Figura 3. Íleon con áreas de disminución de diámetro, serosa con eritema, cambios en el patrón vascular y edema de pared; se visualiza una zona de estenosis fibroestenótica que imposibilita el paso de la cápsula endoscópica.

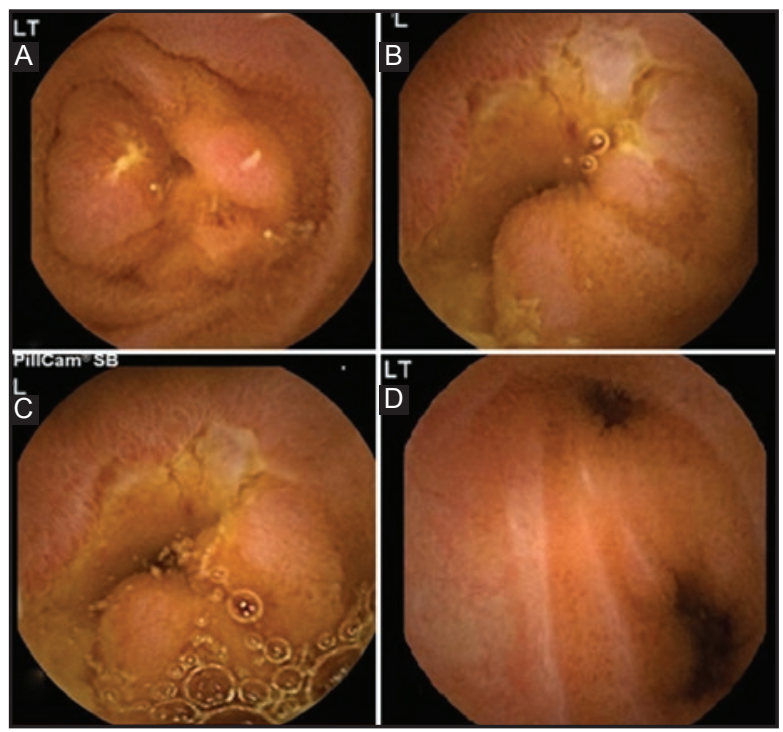

Figura 4. A, B, C: Úlceras detectadas en yeyuno. D: Divertículos en yeyuno.

anastomosis y se egresó al enfermo al sexto día del PO. En el seguimiento PO había evolución adecuada; sin embargo, a las ocho semanas posteriores al acto quirúrgico se presentó salida de material intestinal a través de la pared abdominal. Se sometió a tratamiento médico por fístula intestinal por 18 días con cierre espontáneo de la fístula enterocutánea. El paciente continúa bajo tratamiento médico sin presentar recurrencia de la enfermedad.

\section{Discusión}

En cuanto al enfoque endoscópico, la retención de CE se define como una cápsula retenida en el intestino delgado por dos semanas o más, o menos de ese periodo de tiempo si se requiere tratamiento médico, endoscópico o quirúrgico para la extracción de la $\mathrm{CE}$. En consecuencia, se ha propuesto el término de "impactación" 
para referirse a la CE alojada en una zona estenótica de la luz intestinal que produce obstrucción intestinal total y puede causar perforación intestinal, con una tasa calculada de $0.75 \%{ }^{1,2}$

La mayor parte de los casos de retención de CE se presenta sin ocasionar obstrucción intestinal aguda; en realidad, Cave, et al., mencionan que hasta el 2005 no había notificación en la bibliografía médica de un caso de obstrucción intestinal aguda (OIA) por cápsula endoscópica impactada. ${ }^{1}$ Después de esa fecha se identificaron en las publicaciones siete casos de obstrucción intestinal aguda por retención de $\mathrm{CE}$, de los cuales cuatro se relacionaron con enfermedad de Crohn no diagnosticada. ${ }^{4-7}$

Lin, et al. publicaron en 2006 el caso de una paciente femenina de 71 años de edad con OIA a las 20 horas tras la ingestión de la CE. ${ }^{4}$ De Palma, et al. (2012) informaron dos casos de OIA por CE, uno de ellos un paciente masculino de 60 años de edad con CE impactada en el íleon terminal con perforación intestinal que exigió resección con anastomosis ileocólica. ${ }^{5}$

Sasmal, et al. documentaron en 2015 un caso de una paciente femenina de 57 años de edad con OIA dos días después de la ingestión de la $\mathrm{CE}$, con realización de hemicolectomía derecha e informe de enfermedad de Crohn en el postoperatorio. ${ }^{6}$ En fecha reciente, Sawai, et al. (2018) informaron el caso de una OIA con perforación en íleon a $100 \mathrm{~cm}$ de la válvula ileocecal por CE reabsorbible (patency capsule) en un paciente masculino de 74 años de edad con enfermedad de Crohn. ${ }^{7}$ De estos casos notificados es importante mencionar que los pacientes son mayores de 50 años, al igual que este caso clínico, pero sin diagnóstico preoperatorio de enfermedad de Crohn. El tratamiento quirúrgico no es detallado por lo que la discusión se ve limitada en este aspecto.

Dentro de las recomendaciones disponibles para la prevención de esta complicación figura realizar una prueba de permeabilidad intestinal con estudio contrastado, ya sea mediante enterotomografía, que tiene mayor sensibilidad que el tránsito intestinal, enterorresonancia magnética o una prueba con cápsula degradable (patency capsule), si bien los resultados son aún controvertidos y además no está disponible en este país. Es preciso mencionar que los estudios negativos para obstrucción no excluyen la presencia de estenosis que produzca la retención de la CE en el intestino delgado, dado que $\sim 90 \%$ de los pacientes que presentaron retención de CE contaba con estudios de imagen negativos para obstrucción e incluso no había síntomas de obstrucción. ${ }^{8}$
Desde el punto de vista quirúrgico, se considera que 70 a $90 \%$ de los pacientes con enfermedad de Crohn necesitará al final someterse a algún procedimiento quirúrgico, sea de forma electiva o de urgencia. La obstrucción intestinal es la causa más frecuente de operación en estos pacientes, la cual se acompaña de estenosis. ${ }^{9}$

En relación con la posible utilidad de la CE en la obstrucción intestinal, Cheifetz, et al. señalaron en 2006 que el uso de la cápsula endoscópica en pacientes con sospecha de obstrucción de intestino delgado puede ayudar a la identificación del sitio de obstrucción durante la intervención quirúrgica, a pesar de lo cual estos resultados son muy controvertidos. ${ }^{10}$ En los casos en que se intente la extracción mediante enteroscopia de doble balón con resultado fallido, el marcaje con tinta china permite la pronta identificación del sitio de obstrucción durante la intervención.

Por último, en el tratamiento quirúrgico de la obstrucción intestinal por CE en pacientes con enfermedad de Crohn hay diversos aspectos a considerar para la elección del tipo de operación:

1. El riesgo elevado de fuga de la anastomosis ${ }^{11}$.

2. La utilidad del tratamiento anti-TNF (factor de necrosis tumoral) antes y después de la intervención (no ha demostrado elevar el riesgo de fuga anastomótica ni contraindica la anastomosis primaria) $)^{12-14}$.

3. El tipo de anastomosis a realizar. En este caso, la elección del tipo de anastomosis se basó en un metaanálisis reciente en el que se documentó que la anastomosis laterolateral mecánica se relaciona con menor índice de fuga de la anastomosis y recurrencia quirúrgica ${ }^{15-17}$.

\section{Conclusión}

La retención de la CE es la complicación más grave de este método diagnóstico, ya que puede provocar obstrucción intestinal y perforación intestinal en el peor de los casos. Existen algunos métodos disponibles para disminuir la probabilidad de esta compilación, como el uso de estudios de imagen para valorar la permeabilidad del intestino delgado, aunque la mayoría de los pacientes en los que se ha informado la retención tuvo estudios negativos para obstrucción.

Debido a que se presenta la retención de la CE sin alteración del tránsito intestinal, no hay un tiempo límite para instituir tratamiento y extraer la CE, por lo que esta decisión se basa en los síntomas y condición clínica del paciente. En los casos en que está indicada 
la resección intestinal, se recomienda que sea amplia con anastomosis laterolateral mecánica para reducir el riesgo de recurrencia de la enfermedad.

\section{Conflicto de intereses}

Los autores declaran no tener conflicto de intereses.

\section{Financiamiento}

Los autores aportaron el financiamiento para este trabajo.

\section{Responsabilidades éticas}

Protección de personas y animales. Los autores declaran que los procedimientos seguidos se ajustaron a las normas éticas del comité de experimentación humana responsable y de acuerdo con la Asociación Médica Mundial y la Declaración de Helsinki.

Confidencialidad de los datos. Los autores declaran que han seguido los protocolos de su centro de trabajo sobre la publicación de datos de pacientes.

Derecho a la privacidad y consentimiento informado. Los autores han obtenido el consentimiento informado de los pacientes o sujetos referidos en el artículo. Este documento obra en poder del autor de correspondencia.

\section{Bibliografía}

1. Cave $D$, Legnani $P$, de Franchis $R$, Lewis $S$. ICCE consensus for capsule retention. Endoscopy. 2005;37:1065-7.
2. Rezapour M, Amadi C, Gerson LB. Retention associated with video capsule endoscopy: systematic review and meta-analysis. Gastrointest Endosc. 2017;85:1157-68.

3. Enns RA, Hookey L, Armstrong D, Berstein C, Heitman S, Teshima C, et al. Clinical practice guidelines for the use of video capsule endoscopy. Gastroenterology. 2017;152:497-514.

4. Lin OS, Brandabur JJ, Schembre DB, Soon MS, Kozarek RA. Acute symptomatic small bowel obstruction due to capsule impaction. Gastrointestinal Endoscopy. 2007;65(4):725-728.

5. De Palma GD, Masone S, Persico M, Siciliano S, Salvatori F, Maione F, et al. Capsule impaction presenting as acute small bowel perforation: a case series. J Medical Case Reports. 2012;6:121.

6. Sasmal PK, Das PC, Tantia O, Patle N, Khanna S, Sen B. Acute small intestinal obstruction-an unusual complication of capsule endoscopy. Indian J Surg. 2015;77(Suppl 1):21-23.

7. Sawai K, Goi T, Takegawa Y, Ozaki Y, Taguchi S, Kurebayashi H, et al. Acute small bowel perforation caused by obstruction of a novel tag-less AgileTM patency capsule. Case Rep Gastroenterol. 2018;12(2):337-343.

8. Postgate AJ, Burling D, Gupta A, Fitzpatrick A, Fraser C. Safety, reliability and limitations of the given patency capsule in patients at risk of capsule retention: a 3-year technical review. Dig Dis Sci 2008;53:2732-8.

9. Frolkis AD, Dykeman J, Negrón ME, Debruyn J, Jette N, Fiest KM, et al. Risk of surgery for inflammatory bowel diseases has decreased over time: a systematic review and meta- analysis of population-based studies. Gastroenterology 2013; 145: 996-1006.

10. Cheifetz A, Sachar D, Lewis B. Small bowel obstruction: indication or contraindication for capsule endoscopy. Paper presented at the Digestive Disease Week meeting, May 15-20, 2004, New Orleans, Louisiana, USA.

11. Hyman N, Manchester TL, Osler T, Burns B, Cataldo PA. Anastomotic leaks after intestinal anastomosis: it's later than you think. Ann Surg 2007; 245: 254-258.

12. Myrelid P, Marti-Gallostra M, Ashraf S, Sunde ML, Tholin M, Oresland $\mathrm{T}$, et al. Complications in surgery for Crohn's disease after preoperative antitumour necrosis factor therapy. Br J Surg. 2014; 101: $539-545$.

13. Maguire L, Olariu A, Hicks C, Hodin R, Bordeianou L. Does TNF inhibitor treatment prior to surgery for small bowel crohn's disease modulate disease severity and minimize surgical intervention? Dis Colon Rectum 2014; 57.

14. El-Hussuna A, Andersen J, Bisgaard T, Jess P, Henriksen M, Oehlenschlager $\mathrm{J}$, et al. Biologic treatment or immunomodulation is not associated with postoperative anastomotic complications in abdominal surgery for Crohn's disease. Scandinavian J Gastroenterol 2012; 47: 662-668.

15. Riss S, Bittermann C, Zandl S, Kristo I, Stift A, Papay P, et al. Short-term complications of wide- lumen stapled anastomosis after ileocolic resection for Crohn's disease: who is at risk? Colorectal Dis 2010; 12.

16. He X, Chen Z, Huang J, Lian L, Rouniyar S, Wu X, et al. Stapled side-to-side anastomosis might be better than handsewn end-to- end anastomosis in ileocolic resection for Crohn's disease: a meta- analysis. Dig Dis Sci 2014; 59: 1544-1551.

17. Muñoz-Juárez M, Yamamoto T, Wolff BG, Keighly MRB. Wide-lumen stapled anastomosis vs convencional end-to-end anastomosis in the treatment of Crohn $\square$ s disease. Dis Colon Rectum 2001;44:20-26. 